\title{
Resurgence of malaria in Uganda despite sustained indoor residual spraying and repeated long lasting insecticidal net distributions
}

Adrienne Epstein ${ }^{1,2}$, Catherine Maiteki-Ssebuguzi ${ }^{3}$, Jane F. Namuganga ${ }^{4}$, Joaniter I.

Nankabirwa $^{4,5}$, Samuel Gonahasa ${ }^{4}$, Jimmy Opigo ${ }^{3}$, Sarah G. Staedke ${ }^{6}$, Damian Rutazaana ${ }^{3}$, Emmanuel Arinaitwe ${ }^{4}$, Moses R. Kamya ${ }^{4,5}$, Samir Bhatt ${ }^{7,8}$, Isabel Rodríguez-Barraquer ${ }^{9}$, Bryan Greenhouse $^{9}$, Martin J. Donnelly ${ }^{1}$, Grant Dorsey ${ }^{9}$

${ }^{1}$ Department of Vector Biology, Liverpool School of Tropical Medicine, Liverpool, UK

${ }^{2}$ Department of Epidemiology and Biostatistics, University of California San Francisco, San Francisco, California, United States of America

${ }^{3}$ National Malaria Control Division, Ministry of Health, Kampala, Uganda

${ }^{4}$ Infectious Diseases Research Collaboration, Kampala, Uganda

${ }^{5}$ Makerere University, College of Health Sciences, Kampala, Uganda

${ }^{6}$ London School of Hygiene and Tropical Medicine, London, United Kingdom

${ }^{7}$ Department of Infectious Disease Epidemiology, Imperial College, St Mary's Hospital, London, UK

${ }^{8}$ Section of Epidemiology, Department of Public Health, University of Copenhagen, Copenhagen, Denmark

${ }^{9}$ Department of Medicine, University of California San Francisco, San Francisco, California, United States of America

\section{Corresponding author:}

33 Adrienne Epstein

34 Liverpool School of Tropical Medicine

35 Pembroke Pl, Liverpool L3 5QA

36 Adrienne.Epstein@1stmed.ac.uk 


\section{Abstract}

39 Five years of sustained indoor residual spraying (IRS) of insecticide from 2014 to 2019, first

40 using a carbamate followed by an organophosphate, was associated with a marked reduction in

41 the burden of malaria in five districts of Uganda. We assessed malaria burden over an additional

4221 months, corresponding to a change in IRS formulations using clothianidin with and without

43 deltamethrin and the start of the COVID-19 pandemic. We document an unprecedented

44 resurgence in malaria burden: in years 4-5 of sustained IRS cases were $84 \%$ lower than the pre-

45 IRS period, in year 6 this increased to a $43 \%$ reduction, and in the first 9 months of year 7 , cases

46 were $39 \%$ higher than pre-IRS levels. The timing of this resurgence corresponded to a change of

47 active ingredient to clothianidin, a new IRS active ingredient. Further research is needed to

48 determine mechanisms leading to this resurgence.

49

50 


\section{Introduction}

52 Major gains have been made in reducing the burden of malaria in sub-Saharan Africa over the

53 past two decades, resulting in a 44\% decline in malaria deaths between 2000 and 2019. ${ }^{1,2}$

54 However, progress has slowed in recent years, particularly in highest burden countries. ${ }^{1} \mathrm{~A}$

55 majority of the decline in malaria burden has been attributed to vector control interventions,

56 including long-lasting insecticidal nets (LLINs) and indoor residual spraying of insecticide

57 (IRS). Scale-up of LLINs coverage has occurred rapidly, with the proportion of households in

58 sub-Saharan Africa with at least one LLIN increasing from 5\% in 2000 to $68 \%$ in $2019 .^{1}$

59 Conversely, the percent of at-risk populations covered by IRS has been much lower and even

60 declined from $5 \%$ in 2010 to $2 \%$ in $2019 .{ }^{1}$ Challenges to the scale-up of IRS coverage include

61 high cost, complex implementation logistics, and community acceptance. ${ }^{3}$ Furthermore, evidence

62 from controlled trials is mixed on the added benefit of implementing IRS in communities where

63 LLIN coverage is high. ${ }^{4}$

65 Uganda is illustrative of high burden countries where progress in reducing malaria burden has

66 slowed in recent years. ${ }^{1}$ The Ugandan Ministry of Health has made a strong commitment to

67 ensuring high LLIN coverage, delivering LLINs through 3 universal coverage campaigns (UCC)

68 taking place in 2013-2014, 2017-2018, and 2020-21. These campaigns have been successful: for

69 example, in 2018-2019, 83\% of households reported owning at least one LLIN, the highest

70 coverage globally. ${ }^{5}$ IRS was reintroduced into Uganda in 2006 for the first time since the 1960s,

71 however, geographic coverage of IRS to date has been much lower than LLINs. From 2007 to

72 2014, IRS was implemented in 10 districts in Northern Uganda initially using pyrethroid

73 insecticides and later switching to a carbamate. ${ }^{6}$ In 2014, the IRS campaign was moved to 14 
74 historically high burden districts in North-Eastern Uganda in 2014. The IRS campaign in the

75 northeast initially deployed a carbamate insecticide (2014-2016) before changing to an

76 organophosphate for the third, fourth, and fifth years (2017-2019). In this part of the country,

77 IRS has been considered highly successful: in the first 3 years of sustained IRS campaigns

78 (2014-2017), malaria cases at sentinel surveillance sites in 5 of these districts fell by 50\%, and in

79 the fourth and fifth years (2018-2019), by 85\% compared to pre-IRS levels. ${ }^{7}$

81 In this study, we use data from a network of health facility-based malaria surveillance sites to

82 examine the impact of sustained IRS in the 5 districts mentioned above. This study had two

83 objectives. First (Objective 1), to estimate the impact of IRS on malaria burden in 5 districts

84 relative to a baseline period before IRS was initiated after extending our prior analysis ${ }^{7}$ to the $6^{\text {th }}$

85 and $7^{\text {th }}$ years of sustained IRS (January 2020 through October 2021). This extended evaluation

86 period coincided with a shift in insecticide formulations from the organophosphate pirimiphos-

87 methyl (Actellic 300CS®) to products containing the active ingredient clothianidin alone

88 (SumiShield $\AA$ ) or combined with deltamethrin (Fludora ${ }^{\circledR}$ Fusion WP-SB), the beginning of the

89 COVID-19 pandemic, and the third LLIN UCC (2020-2021). Second (Objective 2), we

90 compared temporal changes in malaria burden during the $6^{\text {th }}$ and $7^{\text {th }}$ years of sustained IRS

91 (January 2020 through October 2021) at the 5 sites receiving sustained IRS to 10 sites in

92 neighboring districts that have not received IRS since 2017.

\section{Results}

\section{$95 \quad$ Study Objective 1}


Across the 5 sites receiving sustained IRS, a total of 769,561 outpatient visits were recorded

97 from the baseline period covering up to 12 months before IRS started through October 2021

98 (Table 1). During the baseline period, average monthly cases adjusted for testing ranged from

99 278-657 and TPR ranged from 25·2\%-67-0\%. By years 4 and 5 (months 37 to 60) of sustained

100 IRS these metrics had decreased to 30-90 and 8.5\%-26.3\%, respectively. However, in year 6 and

101 the first 9 months of year 7 (months 61 to 81) of sustained IRS, average monthly cases adjusted

102 for testing increased to a range of 209-431 and TPR ranged from 27.0\%-52.9\%. Figure 1 shows

103 plots of laboratory-confirmed malaria cases and vector control interventions over time across the

1045 sites. Each of these sites demonstrate similar patterns of a decline in malaria cases after the

105 initiation of IRS with seasonal peaks during the first three years of IRS (through 2017), a

106 substantial decline in burden in years four and five of sustained IRS (through 2019), and an

107 increase in burden in years 6 and 7 of sustained IRS (2020-2021) equal to or higher than the

108 burden in the baseline period before IRS was implemented.

109 Figure 1. Malaria case counts and vector control interventions over time at 5 IRS sites. The 110 study baseline period (pre-IRS) is indicated in grey.
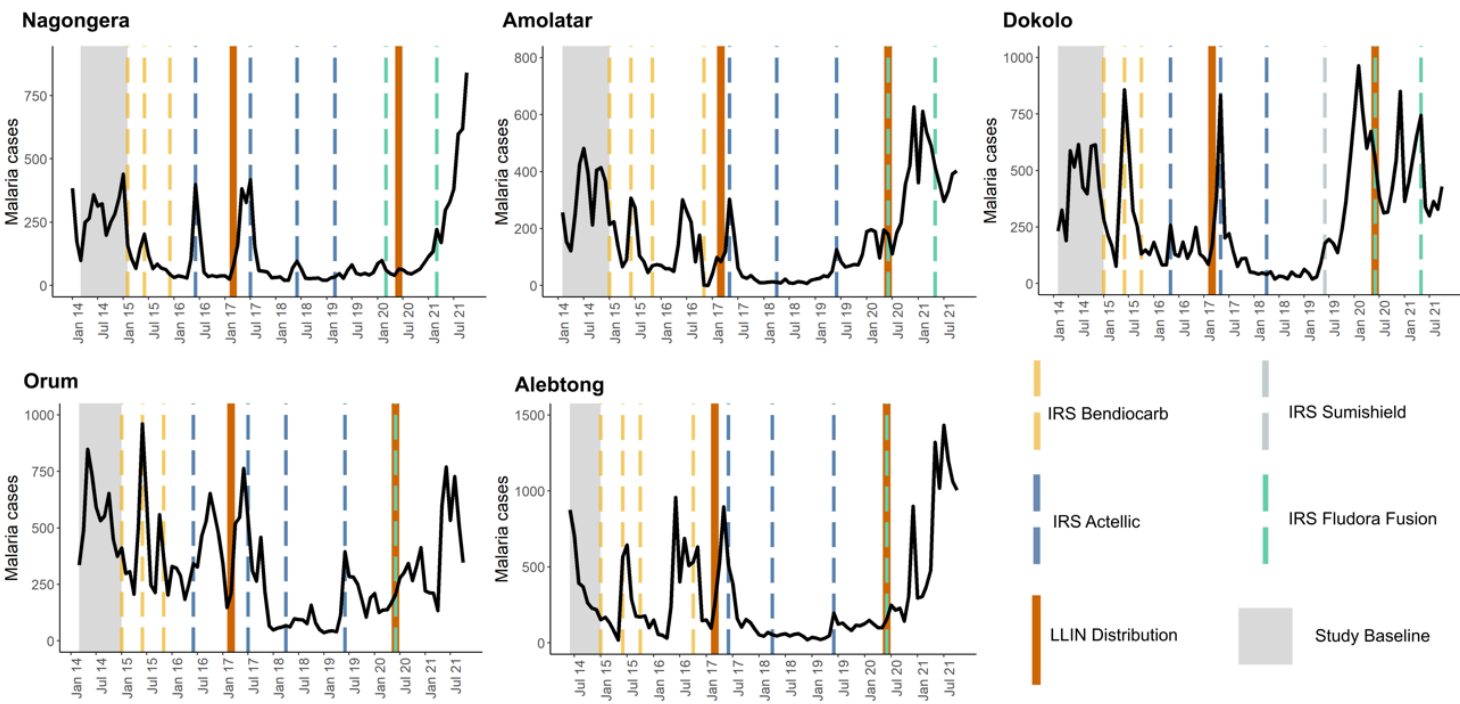
112 Monthly adjusted IRRs and 95\% confidence intervals (CI) for the 5 sites combined are presented

113 in Figure 2 and in Supplemental Table 1. These results show there was an initial 52\% reduction

114 in malaria burden in months 1 through 36 of sustained IRS (adjusted IRR $=0 \cdot 48,95 \%$ CI 0 36-

$1150 \cdot 64$ ), followed by a continued improvement to an $84 \%$ reduction in burden in months 37

116 through 60 of sustained IRS (adjusted IRR =0·16, 95\% CI 0·12-0·22). In months 61 through 72 ,

117 however, malaria burden increased and was only $43 \%$ lower than the baseline period before IRS

118 was implemented (adjusted IRR $=0 \cdot 57,95 \%$ CI 0.44-0·74). During months 73 through 81 after

119 the initiation of IRS, there was a trend towards a higher malaria burden than the pre-IRS period

120 (adjusted IRR $=1 \cdot 39,95 \%$ CI 0.97-1.97). These results were consistent when including only

121 laboratory-confirmed cases unadjusted for testing rate as the model outcome (Supplemental

122 Figure 1) and when repeating the analysis leaving out the 2 sites that halted IRS campaigns after

1232020 (Orum and Alebtong) (Supplemental Figure 2).

124 Figure 2. Adjusted IRR from multilevel negative binomial model comparing the period 125 after IRS was initiated to the period before IRS was initiated. Vertical bars represent the $95 \%$

126 CI around adjusted IRR. Effect estimates in grey are published previously.

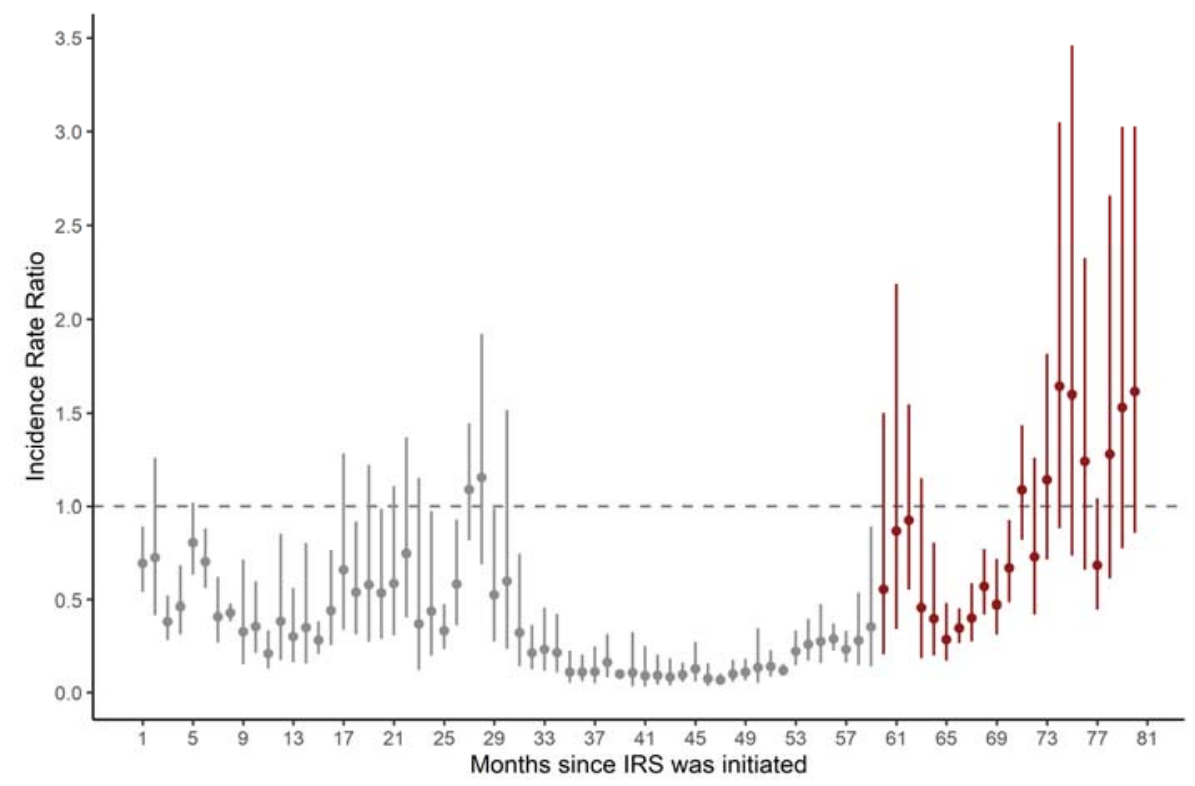




\section{Study Objective 2}

130 Across the 15 sites over a 22 month period (January 2020 through October 2021) included in the

131 analysis for Objective 2, 583,344 outpatient visits were recorded (Table 2). From January 2020

132 through December 2020, average monthly cases at IRS sites ranged from 64-594 and TPR

133 ranged from 10.9\%-51.0\%. From January 2021 through October 2021, these figures increased to

$134 \quad 371-849$ and $43 \cdot 4 \%-60 \cdot 1 \%$, respectively. Average monthly cases increased in 4 of the 5 IRS sites

135 comparing January 2020 through December 2020 to January 2021 through October 2021, with

136 the exception of Dokolo (the first site to switch to IRS with a clothianidin-based formulation in

137 2019), which already experienced a large increase in monthly malaria cases by January 2020.

139 In sites that had not received IRS recently, average monthly cases from January 2020 through

140 December 2020 ranged from 518-1,068 and TPR from 47.5\%-71·1\%. These figures decreased to

$141251-716$ and 32.5\%-50.4\%, respectively, from January 2021 through October 2021. Figure 3

142 shows plots of laboratory-confirmed malaria cases and the timing of the recent LLIN distribution

143 across the 10 non-IRS sites from January 2020 through October 2021 . These figures indicate a

144 substantial increase in cases the first year of observation for some sites, but a general downward

145 trend in cases across the 10 sites over the 22 month study period for Objective 2. 

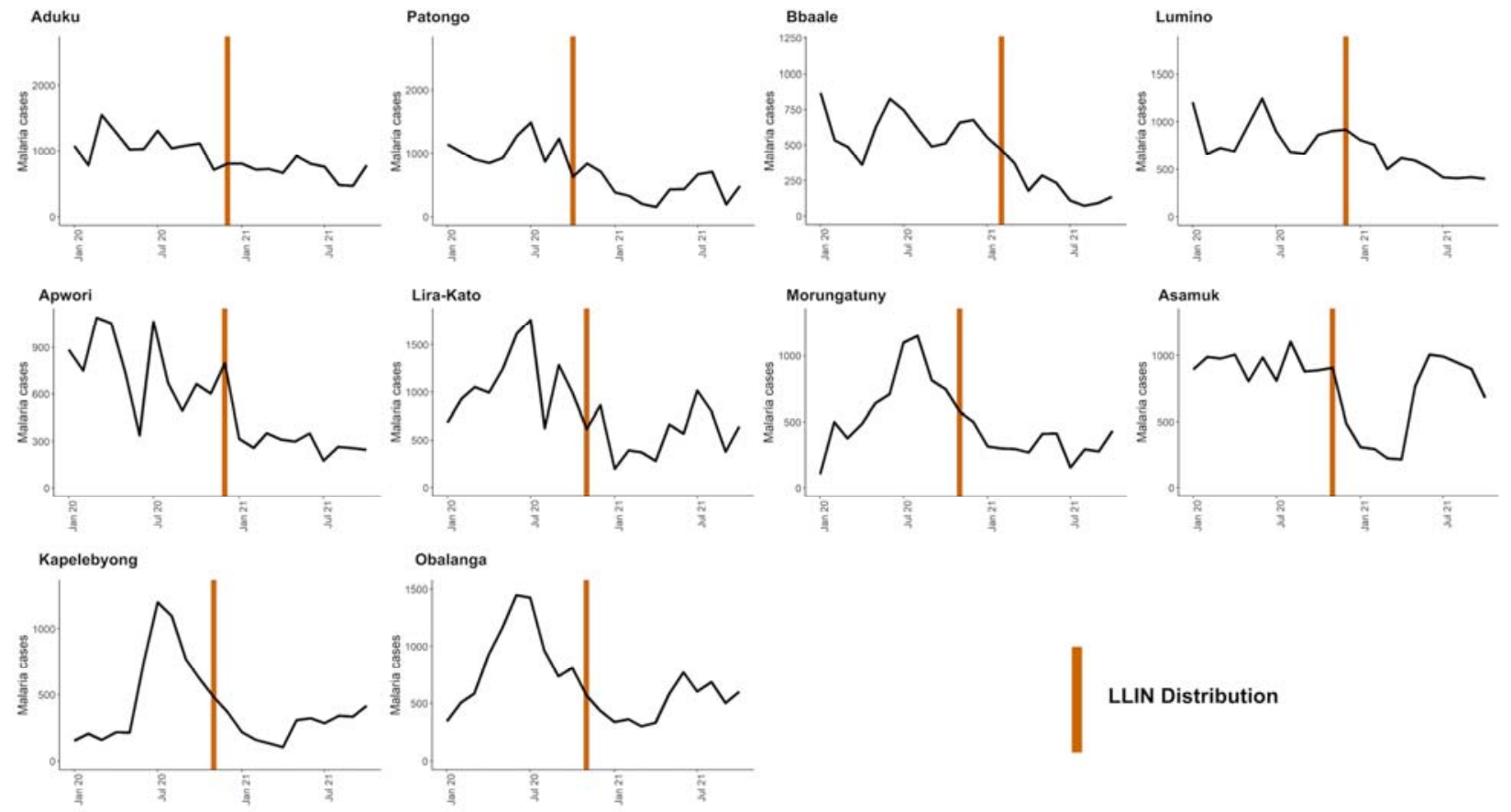

LLIN Distribution

Figure 4 shows the mean number of cases across the 5 IRS sites and the 10 sites that have not received IRS recently from January 2020 to October 2021 (corresponding to months 60 through 81 of IRS for the 5 sites where IRS was initiated and sustained). This figure shows an increase in cases at IRS sites, particularly in the latter half of the observation window, while cases at nonIRS sites trended downward over the same time period. Figure 4 also shows the adjusted IRR comparing cases at IRS sites to sites that have not received IRS recently. Coefficients and 95\% confidence intervals are also presented in Supplemental Table 2. These findings indicate that

157 from January 2020 to November 2020, cases were significantly lower in IRS sites compared to

158 non-IRS sites. From December 2020 onward, the IRR crossed 1, indicating that cases at IRS sites were higher than non-IRS sites, although we could not rule out a null or negative association in 
163 consistent when including only laboratory-confirmed cases unadjusted for testing as the outcome

164 (Supplemental Figure 3).

Figure 4. Mean case counts in 5 IRS sites and 10 non-IRS sites from January 2020 through July 2021 (left) and adjusted IRR comparing IRS sites to non-IRS sites over the study period. Shaded areas represent standard deviations. Vertical bars represent the $95 \%$ CI around 168 adjusted IRR.
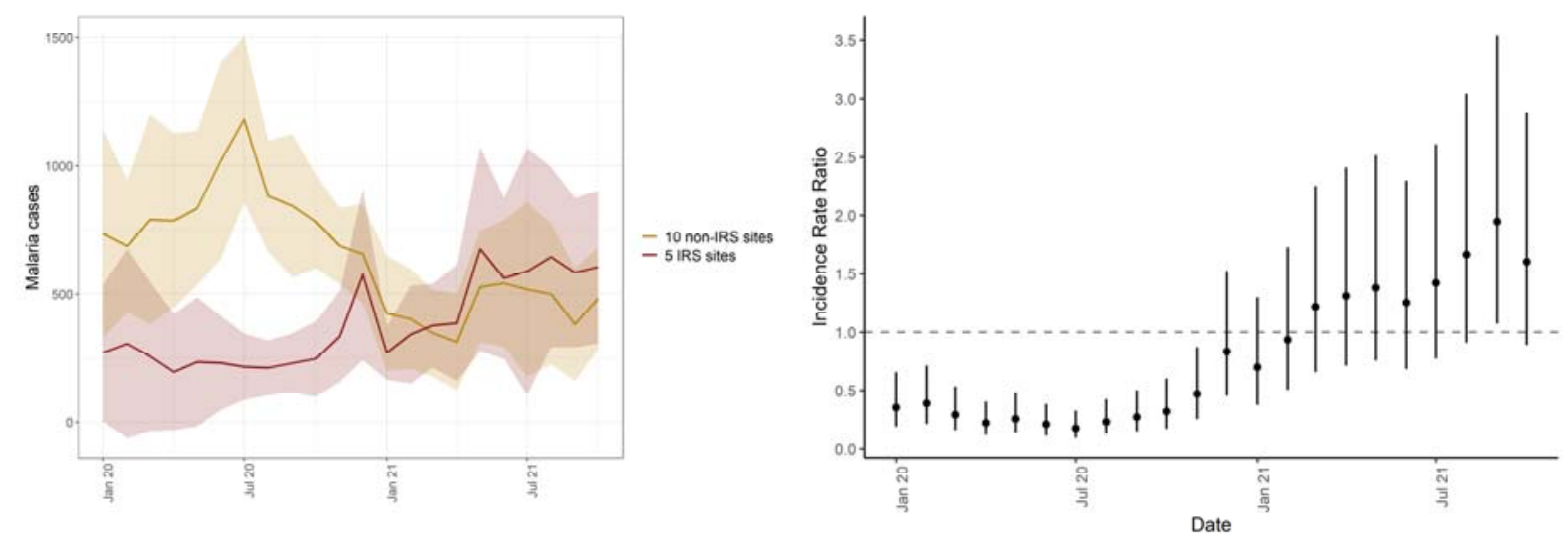

\section{Discussion}

171 We utilized enhanced health facility surveillance data from 5 sites in North-Eastern Uganda that

172 have undergone sustained IRS since 2014 to evaluate the impact of repeated IRS campaigns in

173 their $6^{\text {th }}$ and $7^{\text {th }}$ years. Our findings point to a resurgence in the burden of malaria at these 5

174 health facilities, despite sustained IRS and repeated LLIN UCCs conducted every 3 years. In the

175 final 9 months of observation (months 61-81 of sustained IRS), malaria burden reached similar

176 levels, and in some instances higher levels, than the period before IRS was initiated in late 2014.

177 We did not observe corresponding increases in burden at 10 surveillance sites in neighboring

178 non-IRS districts that, unlike IRS districts, received "next generation" LLINs over the study

179 period. These findings suggest that in the setting of universal coverage with conventional

180 pyrethroid-only LLINs, the marked benefit of adding sustained IRS over the first five years had 
181 been lost over the subsequent 18 months that coincided with a change in active ingredient and

182 the start of the COVID-19 pandemic.

184 This study highlights the importance of high quality routine surveillance to monitor the impact of 185 population level malaria control interventions using clinically relevant indicators such as

186 symptomatic cases diagnosed at health facilities. The assessment of vector control interventions

187 often focuses on entomologic outcomes such as mosquito mortality in controlled settings. This is

188 understandable, given that measuring the impact of control interventions on clinical burden in

189 "real world" settings is difficult. Nevertheless, the findings from this analysis underscore the

190 potential feasibility of and need for robust epidemiologic surveillance systems to document the

191 impact of vector control interventions on population health as they are applied.

193 Routine surveillance in areas undergoing IRS is particularly necessary because published studies

194 from controlled trials on the added value of IRS in areas with high LLIN coverage has produced

195 mixed results. A recent Cochrane review reported that adding IRS using a "pyrethroid-like"

196 insecticide to LLINs did not provide any benefits, while adding IRS with a "non-pyrethroid-like"

197 insecticide produced mixed results. ${ }^{4}$ It is of note that none of the trials that evaluated the impact

198 of adding IRS with a "non-pyrethroid-like" insecticide assessed outcomes beyond two years.

199 More recently, observational studies evaluating the effectiveness of pirimiphos-methyl (Actellic

200 300CS®) in "real world" settings have documented impressive impacts of IRS in Mali",

201 Ghana $^{10}$, Zambia $^{11}$, Kenya ${ }^{12}$, and Uganda. ${ }^{7}$ This analysis contributes to observational data on the

202 impacts of sustained IRS beyond 6 years, in the context of the COVID-19 pandemic and a switch

203 to products containing the active ingredient clothianidin. 
205 There are several potential factors that may be driving the increase in malaria burden in districts

206 receiving IRS. First, the timing of the increase corresponds to a shift in active ingredient from

207 pirimiphos-methyl to clothianidin-based formulations (primarily Fludora® Fusion, a

208 combination of clothianidin and deltamethrin). This change may have led to a partial loss of the

209 combined impact of the dual intervention (combining pirimiphos-methyl to pyrethroid LLINs),

210 which would be particularly detrimental as wide-spread pyrethroid resistance has been described

211 in Uganda. ${ }^{13-15}$ Given that IRS districts received conventional pyrethroid LLINs in the 2020-

2122021 UCC campaign, pyrethroid resistance may also explain why burden increased at IRS sites

213 despite the campaign. Conversely, the 10 sites in neighboring districts where increases in burden

214 were not observed received "next generation" nets designed for areas with wide-spread

215 pyrethroid resistance. Of note, the one site (Dokolo) that received a single round of IRS with

216 clothianidin-based SumiShield ${ }^{\circledR} 50$ WG in 2019 (before the shift to Fludora ${ }^{\circledR}$ Fusion WP-SB

217 across all sites in 2020) experienced an increase in burden immediately following the round of

218 SumiShield ${ }^{8}$ 50WG. Increases were not documented at other sites until the initiation of

219 Fludora ${ }^{\circledR}$ Fusion WP-SB in 2020. This is added evidence for the change in active ingredient

220 contributing to the observed resurgence of malaria. To date, studies on the effectiveness of

221 clothianidin alone (SumiShield $\left.{ }^{\circledR}\right)^{16-20}$ and clothianidin with deltamethrin (Fludora ${ }^{\circledR}$ Fusion WP-

$222 \mathrm{SB})^{16,21-23}$ are limited to susceptibility studies focused on entomological outcomes, both in lab

223 and in experimental huts. These studies document that clothianidin-based products succeed at

224 killing mosquitoes, but none have evaluated clinically relevant outcomes in "real world" settings. 
226 A second potential contributor to the observed resurgence of malaria at sites receiving IRS is the

227 COVID-19 pandemic. An important concern has been the potential for delayed or inadequate

228 implementation of vector control measures due to the pandemic. ${ }^{24,25}$ In Uganda, the LLIN

229 distribution campaign was delayed by 5 months but successfully distributed over 28 million nets,

230 achieving over $90 \%$ coverage. Given that the delay in the UCC was country-wide, we do not

231 believe an increase in burden resulting from delayed net distribution would be observed strictly

232 in IRS sites as was the case in this study. The implementation of IRS campaigns did not appear

233 impacted by the COVID-19 pandemic; annual campaigns in 2020 and 2021 were not delayed and

234 coverage remained high (>90\%, see Supplemental Table 3). We cannot rule out the potential for

235 undocumented differences in implementation (for example, if spray operators spent less time in

236 homes due to fear of acquiring COVID-19) that may have contributed to the observed

237 resurgence. The pandemic may also have impacted patient behavior; for example, patients may

238 have delayed seeking care for malarial illness which could have led to increased transmission.

239 However, because sites that have not recently received IRS did not observe an increase in

240 transmission, this explanation is less tenable. Furthermore, an analysis assessing the potential

241 impact of the first year of the COVID-19 pandemic at Uganda Malaria Surveillance Program

242 (UMSP) sites found no impact of the pandemic on malaria cases and non-malarial visits at health

243 facilities. $^{26}$

245 Another potential explanation is a shift in mosquito species composition or mosquito behavior to

246 outdoor biting, circumventing vector control interventions that target indoor biting mosquitoes. ${ }^{27}$

247 This, however, would be unlikely to have occurred rapidly and simultaneously at all IRS sites at

248 a pace that would explain the resurgence observed in this study. In addition, this would not 
explain the observation that the resurgence was observed only in the IRS sites and not in the non-

250 IRS sites that only received LLINs, as a shift to outdoor biting would have a negative impact on

251 both IRS and LLINs. Similarly, a shift in the predominant species from Anopheles gambiae and

252 Anopheles funestus to Anopheles arabiensis may have led to a change from predominately

253 indoor to outdoor biting. ${ }^{28}$ Recent data demonstrate that Anopheles gambiae and Anopheles

254 funestus remain the primary vectors in $\operatorname{Uganda}^{15}$, but other work has documented the near

255 collapse of Anopheles gambiae and Anopheles funestus in areas with an LLIN distribution and

256 repeated IRS campaigns. ${ }^{28}$

258 This study is not without limitations. First, we used an observational study design, with measures

259 of impact based on comparisons made before-and-after the implementation of IRS and

260 comparisons of IRS and non-IRS districts. While cluster randomized controlled trials remain the

261 gold standard design for estimating the impact of IRS, withholding IRS may be unethical, given

262 what is known about its beneficial impacts, particularly in Uganda. ${ }^{7}$ Similarly, comparisons of

263 IRS sites and sites that have not recently received IRS are strictly descriptive, given that districts

264 receiving IRS and not receiving IRS are not exchangeable. Second, while we hypothesize

265 potential mechanisms that may explain the observed resurgence in malaria burden in IRS sites,

266 we cannot rule potential secular trends or other unmeasured contributing factors. For example,

267 while we attempt to control for seasonal effects and climate-related drivers of malaria burden

268 over time, it is certainly possible that more recent months over the observation period correspond

269 to an unusually high burden period in Uganda. However, overall secular trends are an unlikely

270 cause given the contemporaneous decline in malaria burden at nearby non-IRS sites. Third, the

271 outcome for this analysis is limited to case counts of laboratory-confirmed malaria captured at 
272 health facilities. We do not have additional data on other metrics of transmission intensity,

273 including entomologic measures, residual efficacy, nor on malaria mortality.

275 Despite these limitations, this analysis has important scientific and policy implications. First,

276 additional research is needed on the driving factors contributing to the observed resurgence of

277 malaria burden in IRS districts, including targeted entomology and residual efficacy.

278 Furthermore, the Ministry of Health should be prepared to make timely changes in malaria

279 control interventions based on on-going surveillance. For example, in IRS districts, policy

280 makers may consider switching to the use of newer generation LLINs containing PBO which

281 have been shown to be more effective than traditional pyrethroid treated LLINs in Uganda. ${ }^{29}$

282 Future changes to the IRS active ingredient should take into consideration on-going surveillance,

283 in line with Uganda's resistance management strategy. Consideration should also be made to key

284 logistical factors including cost, procurement, and community acceptability. The unprecedented

285 increase in malaria burden in areas where incidence had declined by $85 \%$ underscores the need

286 to remain vigilant and responsive. Indeed, two of the five IRS districts included in this analysis

287 stopped receiving IRS altogether in 2021, highlighting the challenge of maintaining gains in the

288 face of inadequate resources and the need for rational exit strategies when IRS cannot be

289 sustained. Finally, maintaining high quality, continuous surveillance systems to assess the impact

290 of population level malaria control interventions remains essential in order to generate timely,

291 actionable data.

292

293 Methods

294 Study sites and vector control interventions 
295 This study utilized enhanced health facility surveillance data from 15 health facilities in Uganda.

296 Five of these health facilities are among the 14 districts that have received repeated rounds of

297 IRS since December 2014 (the remaining 9 districts did not have surveillance sites). Ten health

298 facilities are in neighboring districts that have never received IRS (6 sites) or where IRS was last

299 implemented in 2017 (4 sites, Figure 5). All facilities included in the analysis are part of a

300 surveillance network called the UMSP. National LLIN UCCs were conducted in 2013-2014,

301 2017-2018, and 2020-2021, where LLINs were distributed free-of-charge by the Uganda

302 Ministry of Health targeting one LLIN for every two household residents throughout the entire

303 country (following WHO recommendations). During the most recent campaign (2020-2021), the

304 Ministry of Health distributed conventional LLINs containing pyrethroid in addition to two types

305 of "next generation" LLINs due to evidence of pyrethroid resistance: one containing

306 deltamethrin and piperonyl butoxide (PBO; Permanet $\left.{ }^{\circledR} 3 \cdot 0\right)$ and one containing alpha-

307 cypermethrin and pyriproxyfen (Royal Guard®). Among the surveillance sites included in this

308 study, the 5 sites in IRS districts were in sub-counties that received conventional pyrethroid nets

309 and the 10 sites in neighboring districts were in sub-counties that received "next generation" nets

310 (5 received Permanet ${ }^{\circledR} 3 \cdot 0$ nets and 5 received Royal Guard® nets). 
Figure 5. Map of Uganda showing study sites.

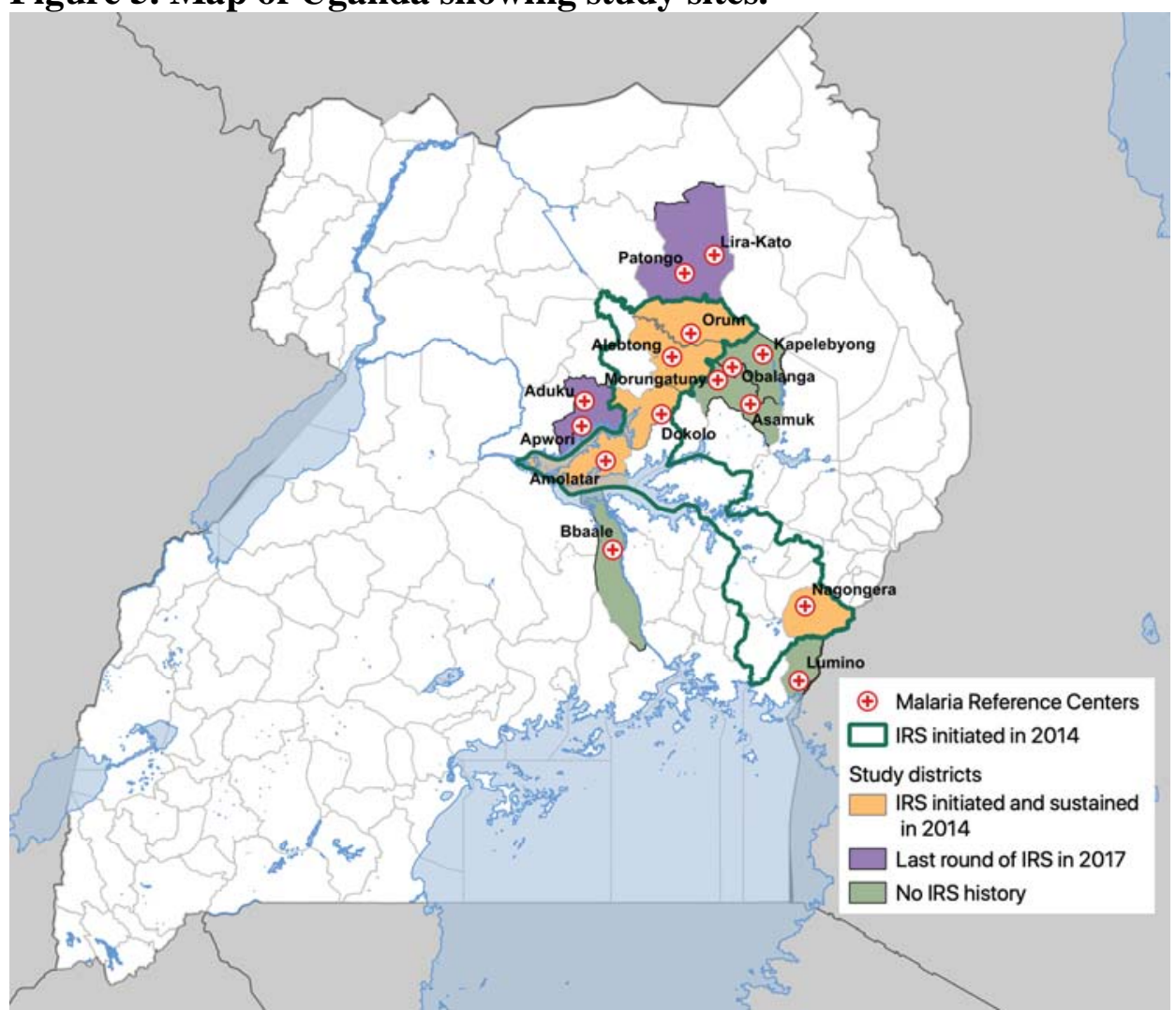

314 In the 5 study districts where IRS was first implemented in late 2014, the insecticide formulation

315 initially consisted of a carbamate (bendiocarb) with rounds repeated approximately every 6

316 months until 2016 when the active ingredient was changed to the organophosphate pirimiphos-

317 methyl (Actellic 300CS®) administered annually until 2019. In 2019, one study district (Dokolo)

318 received a single round of IRS with SumiShield® 50WG, a new IRS product containing

319 clothianidin. In 2020, all IRS districts began receiving IRS with Fludora® Fusion WP-SB, a new

320 IRS insecticide containing a mixture of clothianidin and deltamethrin. Changes of IRS

321 insecticides were made in accordance with the Ugandan Ministry of Health's insecticide

322 resistance management plan which, in line with WHO recommendations, requires changing IRS

323 formulations every three years to preempt the development of resistance. ${ }^{30,31}$ In two study

324 districts (Otuke and Alebtong), IRS was discontinued in 2021 due to lack of funding. For a 
complete timeline of all IRS campaigns, including insecticides and coverage, see Supplemental

326 Table 3.

\section{Health-facility based surveillance}

329 A network of enhanced malaria surveillance sites embedded in public health facilities was

330 established by UMSP in 2006, as previously described. ${ }^{32}$ In brief, UMSP currently operates

331 Malaria Reference Centers (MRCs) at 70 level III/IV public health facilities across Uganda.

332 Level III and IV health facilities are parish and sub-county level facilities, respectively, and

333 provide diagnostic testing and treatment free of charge to populations of 20,000 to 100,000

334 people per facility. At MRCs, individual-level data are collected for all outpatients using

335 outpatient registers (HMIS 002) and entered monthly into a database by on-site data entry

336 officers. Information collected includes sociodemographic data (age, sex, and village of

337 residence), whether malaria was suspected, results of laboratory testing for malaria (rapid

338 diagnostic test [RDT] or microscopy), diagnoses (laboratory-confirmed and clinical), and

339 treatments prescribed. UMSP places emphasis on high quality data, ensuring minimal

340 missingness on key variables including age and place of residence, and providing training and

341 materials to maximize diagnostic testing among patients suspected of having malaria. For

342 Objective 1 (to evaluate the impact of IRS on malaria burden in 5 districts over $6 \cdot 75$ of sustained

343 use), we utilized data from 5 MRCs from districts with where IRS was implemented in 2014.

344 These MRCs were selected because they had been active UMSP sites for at least 6 months prior

345 to the initiation of IRS. To compare changes in malaria burden from January 2020 through

346 October 2021 (years 6 \& 7 following implementation of IRS) at sites receiving sustained IRS to

347 sites in neighboring districts that have not received IRS recently, we used data from 10 additional 
348 MRCs. These sites were selected because they were in districts that neighbor the 5 IRS sites and

349 have been active UMSP sites since at least January 2020. Four of these sites were in 2 districts

350 that received IRS in the past; however, IRS was last implemented in 2017. The impact of these

351 previous IRS campaigns was no longer evident during the study period. ${ }^{7}$

\section{Measures}

354 For Objective 1, the exposure was specified as an indicator variable representing each month

355 since IRS was initiated. In separate models, an indicator variable, representing months 1-36

356 (years 1, 2, and 3), 37-60 (years 4 and 5), 61-72 (year 6), and 73-81 (the first 9 months of year 7)

357 of sustained IRS was included as the primary exposure variable. The baseline period was defined

358 as the 12 months before IRS was implemented in 2014; if a site had less than 12 months of

359 baseline data available, we included the maximum amount of time available.

361 For Objective 2, the primary exposure was specified as a binary variable representing whether a

362 site was an IRS site or a non-IRS site.

364 For both objectives, the primary outcome was the monthly count of laboratory-confirmed

365 malaria cases at each MRC. To correct for monthly testing rates, we adjusted this count by

366 multiplying the number of individuals with suspected malaria but not tested each month by the

367 test positivity rate (TPR, the number who tested positive divided by the total number tested) in

368 that month. We then added the result to the number of laboratory-confirmed positive cases in that

369 month. As a sensitivity analysis, we re-specified the models including only laboratory-confirmed

370 case counts as the outcome. 
372 We adjusted for time-varying variables that impact malaria burden and malaria case detection at

373 the health facility. This includes monthly precipitation ${ }^{33}$ which was modeled non-linearly using

374 restricted cubic splines. Lags of $0,1,2$, and 3 months were considered for precipitation; the

375 appropriate lag was selected by running univariable regressions with each lag and selecting that

376 which demonstrated the lowest Akaike's information criterion (AIC). We also included indicator

377 variables for month of the year (to adjust for season), the proportion of tests that were RDT

378 (vs. microscopy) in that month, and the number of individuals who attended the health facility

379 but were not suspected of having malaria in that month (to adjust for potential changes in care-

380 seeking behaviors, particularly during the COVID-19 lockdown in Uganda).

\section{Statistical analysis}

383 For Objective 1, we specified mixed effects negative binomial regression models with random

384 intercepts for health facility. ${ }^{7}$ Coefficients for the exposure variable were exponentiated to

385 represent the incidence rate ratio (IRR) comparing the incidence of malaria in the month of

386 interest relative to the baseline pre-IRS period. These models test the null hypothesis of no

387 difference of changes in IRS burden after the initiation of IRS compared to before the initiation

388 of IRS, adjusting for seasonal effects and time-varying changes in diagnostic testing and care

389 seeking.

391 For Objective 2, models were specified as mixed effects negative binomial regression models

392 with random intercepts for health facility. The binary indicator variable representing whether a

393 site was an IRS site or a non-IRS site was interacted with an indicator variable representing time 
394 (an indicator representing month/year and, in a separate model, a categorical variable

395 representing January 2020-December 2020 and January 2021-October 2021). Coefficients for the

396 exposure variable combined with the interaction term were exponentiated to represent the

397 incidence rate ratio (IRR) comparing the burden of malaria IRS sites versus non-IRS sites over a

398 given period of time. These models test the null hypothesis of no difference between malaria

399 burden at IRS sites compared to non-IRS sites, adjusting for seasonal effects and time-varying

400 changes in diagnostic testing and care seeking.

402 Data availability

403 The datasets generated during and/or analysed during the current study are available in GitHub at

404 the URL https://github.com/aeepstein/resurgence-irs-uganda 


\section{Acknowledgements}

407 We would like to acknowledge the health workers at the health facilities that contributed data for

408 this study. We would like to thank the Ugandan Ministry of Health National Malaria Control

409 Division, and USAID - President's Malaria Initiative. This work was supported by the National

410 Institutes of Health as part of the International Centers of Excellence in Malaria Research

411 (ICMER) program (U19AI089674). Funding for this work was also supported by the National

412 Institute of Allergy and Infectious Diseases (F31AI150029).

\section{Author contributions}

416 AE and GD conceived of the study. JFN led the data collection activities with support from

417 CMS, JIN, EA, and SG. AE led the data analysis with support from GD, SB, IRB, and BG. AE

418 and GD drafted the manuscript with support from CMS, JFN, SGS, IRB, BG, MRK, and MJD.

419 All authors contributed to interpretation of the results and edited the manuscript. All authors read 420 and approved the final manuscript.

\section{Competing Interests}

423 The authors declare no competing interests. 
4271 World malaria report 2020: 20 years of global progress and challenges. (World Health $428 \quad$ Organization, Geneva, 2020).

4292 Bhatt, S. et al. The effect of malaria control on Plasmodium falciparum in Africa between 4302000 and 2015. Nature 526, 207-211, doi:10.1038/nature15535 (2015).

4313 Sherrard-Smith, E. et al. Systematic review of indoor residual spray efficacy and 432 effectiveness against Plasmodium falciparum in Africa. Nat Commun 9, 4982, 433 doi:10.1038/s41467-018-07357-w (2018).

4344 Choi, L., Pryce, J. \& Garner, P. Indoor residual spraying for preventing malaria in 435 communities using insecticide-treated nets. Cochrane Database Syst Rev 5, CD012688, 436 doi:10.1002/14651858.CD012688.pub2 (2019).

4375 Uganda National Malaria Control Division (NMCD), Uganda Bureau of Statistics 438 (UBOS) \& ICF. Uganda Malaria Indicator Survey 2018-19. (NMCD, UBOS, and ICF, 439 Kampala, Uganda, and Rockville, Maryland, USA, 2020).

4406 Raouf, S. et al. Resurgence of Malaria Following Discontinuation of Indoor Residual 441 Spraying of Insecticide in an Area of Uganda With Previously High-Transmission 442 Intensity. Clin Infect Dis 65, 453-460, doi:10.1093/cid/cix251 (2017).

4437 Namuganga, J. F. et al. The impact of stopping and starting indoor residual spraying on 444 malaria burden in Uganda. Nat Commun 12, 2635, doi:10.1038/s41467-021-22896-5 $445 \quad(2021)$. 
4468 Wagman, J. et al. Rapid reduction of malaria transmission following the introduction of

447 indoor residual spraying in previously unsprayed districts: an observational analysis of

448 Mopti Region, Mali, in 2017. Malar J 19, 340, doi:10.1186/s12936-020-03414-2 (2020).

$4499 \quad$ Kane, F. et al. Performance of IRS on malaria prevalence and incidence using

450 pirimiphos-methyl in the context of pyrethroid resistance in Koulikoro region, Mali.

451 Malar J 19, 286, doi:10.1186/s12936-020-03357-8 (2020).

45210 Gogue, C. et al. An observational analysis of the impact of indoor residual spraying in

453 Northern, Upper East, and Upper West Regions of Ghana: 2014 through 2017. Malar J

$454 \quad$ 19, 242, doi:10.1186/s12936-020-03318-1 (2020).

45511 Hast, M. A. et al. The Impact of 3 Years of Targeted Indoor Residual Spraying With

456 Pirimiphos-Methyl on Malaria Parasite Prevalence in a High-Transmission Area of

457 Northern Zambia. Am J Epidemiol 188, 2120-2130, doi:10.1093/aje/kwz107 (2019).

45812 Abong'o, B. et al. Impact of indoor residual spraying with pirimiphos-methyl (Actellic

459 300CS) on entomological indicators of transmission and malaria case burden in Migori

460 County, western Kenya. Sci Rep 10, 4518, doi:10.1038/s41598-020-61350-2 (2020).

46113 Tchouakui, M. et al. Pyrethroid Resistance Aggravation in Ugandan Malaria Vectors Is

462 Reducing Bednet Efficacy. Pathogens 10, doi:10.3390/pathogens 10040415 (2021).

46314 Okia, M. et al. Insecticide resistance status of the malaria mosquitoes: Anopheles

464 gambiae and Anopheles funestus in eastern and northern Uganda. Malar J 17, 157,

465 doi:10.1186/s12936-018-2293-6 (2018).

46615 Lynd, A. et al. LLIN Evaluation in Uganda Project (LLINEUP): a cross-sectional survey

467 of species diversity and insecticide resistance in 48 districts of Uganda. Parasit Vectors

$468 \quad$ 12, 94, doi:10.1186/s13071-019-3353-7 (2019). 
46916 Oxborough, R. M. et al. Susceptibility testing of Anopheles malaria vectors with the

470 neonicotinoid insecticide clothianidin; results from 16 African countries, in preparation

471 for indoor residual spraying with new insecticide formulations. Malar J 18, 264,

472 doi:10.1186/s12936-019-2888-6 (2019).

47317 Marti-Soler, H. et al. Effect of wall type, delayed mortality and mosquito age on the

474 residual efficacy of a clothianidin-based indoor residual spray formulation (SumiShield

475 50WG) in southern Mozambique. PLoS One 16, e0248604,

476 doi:10.1371/journal.pone.0248604 (2021).

47718 Agossa, F. R. et al. Efficacy of a novel mode of action of an indoor residual spraying

478 product, SumiShield(R) 50WG against susceptible and resistant populations of

479 Anopheles gambiae (s.1.) in Benin, West Africa. Parasit Vectors 11, 293,

480 doi:10.1186/s13071-018-2869-6 (2018).

48119 Ngwej, L. M. et al. Indoor residual spray bio-efficacy and residual activity of a

482 clothianidin-based formulation (SumiShield((R)) 50WG) provides long persistence on

483 various wall surfaces for malaria control in the Democratic Republic of the Congo. Malar

$484 \quad J$ 18, 72, doi:10.1186/s12936-019-2710-5 (2019).

$48520 \quad$ Kweka, E. et al. Novel Indoor Residual Spray Insecticide With Extended Mortality

486 Effect: A Case of SumiShield 50WG Against Wild Resistant Populations of Anopheles

487 arabiensis in Northern Tanzania. Glob Health Sci Pract 6, 758-765, doi:10.9745/GHSP-

488 D-18-00213 (2018).

48921 Fongnikin, A. et al. Efficacy of Fludora(R) Fusion (a mixture of deltamethrin and

490 clothianidin) for indoor residual spraying against pyrethroid-resistant malaria vectors: 
laboratory and experimental hut evaluation. Parasit Vectors 13, 466, doi:10.1186/s13071-020-04341-6 (2020).

49322 Fuseini, G., Phiri, W. P., von Fricken, M. E., Smith, J. \& Garcia, G. A. Evaluation of the residual effectiveness of Fludora fusion WP-SB, a combination of clothianidin and deltamethrin, for the control of pyrethroid-resistant malaria vectors on Bioko Island, Equatorial Guinea. Acta Trop 196, 42-47, doi:10.1016/j.actatropica.2019.05.006 (2019). Agossa, F. R. et al. Small-scale field evaluation of the efficacy and residual effect of Fludora((R)) Fusion (mixture of clothianidin and deltamethrin) against susceptible and resistant Anopheles gambiae populations from Benin, West Africa. Malar J 17, 484, doi:10.1186/s12936-018-2633-6 (2018).

50124 Seelig, F. et al. The COVID-19 pandemic should not derail global vector control efforts. PLoS Negl Trop Dis 14, e0008606, doi:10.1371/journal.pntd.0008606 (2020).

50325 Guerra, C. A. et al. Malaria vector control in sub-Saharan Africa in the time of COVID(2020).

50626 Namuganga, J. F. et al. Impact of COVID-19 on routine malaria indicators in rural 04018-0 (2021).

50927 Lindsay, S. W., Thomas, M. B. \& Kleinschmidt, I. Threats to the effectiveness of 510 insecticide-treated bednets for malaria control: thinking beyond insecticide resistance. Lancet Glob Health 9, e1325-e1331, doi:10.1016/S2214-109X(21)00216-3 (2021). 
51228 Mawejje, H. D. et al. Impact of seasonality and malaria control interventions on

513 Anopheles density and species composition from three areas of Uganda with differing

514 malaria endemicity. Malar J 20, 138, doi:10.1186/s12936-021-03675-5 (2021).

51529 Staedke, S. G. et al. Effect of long-lasting insecticidal nets with and without piperonyl

516 butoxide on malaria indicators in Uganda (LLINEUP): a pragmatic, cluster-randomised

517 trial embedded in a national LLIN distribution campaign. Lancet 395, 1292-1303,

518 doi:10.1016/S0140-6736(20)30214-2 (2020).

$51930 \quad$ WHO. Global Plan for Inescticide Resistance Management in Malaria Vectors. (2012).

52031 Ministry of Health. The Uganda Malaria Reduction Strategic Plan 2014-2020. (Kampala,

$521 \quad$ Uganda, 2014).

52232 Sserwanga, A. et al. Improved malaria case management through the implementation of a 523 health facility-based sentinel site surveillance system in Uganda. PLoS One 6, e16316, 524 doi:10.1371/journal.pone.0016316(2011).

52533 Funk, C. et al. The climate hazards infrared precipitation with stations--a new 526 environmental record for monitoring extremes. Sci Data 2, 150066, 527 doi:10.1038/sdata.2015.66 (2015). 


\begin{tabular}{|c|c|c|c|c|c|c|c|c|}
\hline MRC (District) & Time period & $\begin{array}{c}\text { Total } \\
\text { outpatient } \\
\text { visits, } \mathrm{n}\end{array}$ & $\begin{array}{l}\text { Suspected malaria } \\
\text { cases, } n \text { (\% of } \\
\text { outpatient visits) }\end{array}$ & $\begin{array}{l}\text { Tested for malaria, } \\
\mathrm{n} \text { (\% of suspected } \\
\text { malaria cases) }\end{array}$ & $\begin{array}{l}\text { RDT performed } \\
\text { (versus } \\
\text { microscopy), } \mathrm{n} \text { (\% } \\
\text { of tested) }\end{array}$ & $\begin{array}{c}\text { Confirmed malaria } \\
\text { cases, } n(\% \text { of } \\
\text { tested [TPR]) }\end{array}$ & $\begin{array}{l}\text { Confirmed malaria } \\
\text { cases adjusted for } \\
\text { testing }\end{array}$ & $\begin{array}{c}\text { Mean monthly } \\
\text { malaria cases } \\
\text { adjusted for testing }\end{array}$ \\
\hline \multirow{4}{*}{$\begin{array}{l}\text { Nagongera HCIV } \\
\text { (Tororo) }\end{array}$} & Baseline (12 months pre-IRS) & 20,828 & $13,251(63 \cdot 6)$ & 13,096 (98.8) & $760(5 \cdot 8)$ & $3,298(25 \cdot 2)$ & 3,337 & 278 \\
\hline & Months $1-36$ of IRS & 63,289 & $23,315(38 \cdot 4)$ & $24,084(99.0)$ & $9,615(40 \cdot 0)$ & $4,004(16 \cdot 6)$ & 4,042 & 112 \\
\hline & Months $37-60$ of IRS & 35,745 & $12,616(35 \cdot 2)$ & $12,608(99 \cdot 9)$ & $3,798(30 \cdot 1)$ & $1,067(8 \cdot 5)$ & 1,067 & 30 \\
\hline & Months $61-81$ of IRS & 38,462 & $16,237(42 \cdot 2)$ & $16,233(99 \cdot 9)$ & $12,219(75 \cdot 3)$ & $4,386(27 \cdot 0)$ & 4,386 & 209 \\
\hline \multirow{4}{*}{$\begin{array}{l}\text { Amolatar HCIV } \\
\text { (Amolatar) }\end{array}$} & Baseline (12 months pre-IRS) & 19,552 & $8,547(43 \cdot 7)$ & $6,512(76 \cdot 2)$ & $5,923(91 \cdot 0)$ & $3,701(56 \cdot 8)$ & 4,845 & 404 \\
\hline & Months $1-36$ of IRS & 55,570 & $18,118(32 \cdot 6)$ & 15,082 (83.2) & $13,440(89 \cdot 1)$ & $3,924(26 \cdot 0)$ & 4,956 & 138 \\
\hline & Months $37-60$ of IRS & 35,231 & $7,038(20 \cdot 0)$ & $7,034(99.9)$ & $6,279(89 \cdot 3)$ & $908(12.9)$ & 1,088 & 30 \\
\hline & Months $61-81$ of IRS & 34,512 & $13,211(38 \cdot 3)$ & $13,180(99 \cdot 8)$ & $11,176(84 \cdot 8)$ & $6,974(52 \cdot 9)$ & 6,992 & 332 \\
\hline \multirow{4}{*}{$\begin{array}{l}\text { Dokolo HCIV } \\
\text { (Dokolo) }\end{array}$} & Baseline (12 months pre-IRS) & 25,570 & $12,854(50 \cdot 3)$ & $8,875(69 \cdot 0)$ & $8,212(92 \cdot 5)$ & $5,211(58 \cdot 7)$ & 7,889 & 657 \\
\hline & Months $1-36$ of IRS & 78,969 & $30,846(39 \cdot 1)$ & $29,476(95 \cdot 6)$ & $27006(91.6)$ & $7,734(26 \cdot 2)$ & 8,266 & 230 \\
\hline & Months $37-60$ of IRS & 52,550 & $16,361(31 \cdot 1)$ & $16,273(99 \cdot 5)$ & $15,997(98 \cdot 3)$ & $2,524(15 \cdot 6)$ & 3,243 & 90 \\
\hline & Months $61-81$ of IRS & 54,489 & $23,142(42 \cdot 5)$ & $23,084(99 \cdot 8)$ & $20,671(89 \cdot 5)$ & $10,887(47 \cdot 2)$ & 10,913 & 520 \\
\hline \multirow{4}{*}{$\begin{array}{c}\text { Orum HCIV } \\
\text { (Otuke) }\end{array}$} & Baseline (11 months pre-IRS) & 16,120 & $9,324(57 \cdot 8)$ & $8,929(95 \cdot 8)$ & $3,990(44 \cdot 7)$ & $5,974(66 \cdot 9)$ & 6,236 & 566 \\
\hline & Months $1-36$ of IRS & 42632 & $26,642(62 \cdot 5)$ & $25,583(96 \cdot 0)$ & $11008(43 \cdot 0)$ & $13,619(53 \cdot 2)$ & 14,207 & 394 \\
\hline & Months $37-60$ of IRS & 23,424 & $11,064(47 \cdot 2)$ & $11,064(100 \cdot 0)$ & $8,717(78.9)$ & $2,911(26 \cdot 3)$ & 3,072 & 85 \\
\hline & Months $61-81$ of IRS & 21,092 & $13,875(65 \cdot 8)$ & $13,875(100 \cdot 0)$ & $8,287(59 \cdot 7)$ & $6,979(50 \cdot 3)$ & 6,979 & 332 \\
\hline \multirow{4}{*}{$\begin{array}{l}\text { Alebtong HCIV } \\
\text { (Alebtong) }\end{array}$} & Baseline (8 months pre-IRS) & 15,359 & $6,694(43 \cdot 6)$ & $4,789(71.5)$ & $4,620(96 \cdot 5)$ & $3,209(67 \cdot 0)$ & 4,317 & 540 \\
\hline & Months $1-36$ of IRS & 62,161 & $30,226(48 \cdot 6)$ & $25,863(85 \cdot 6)$ & $22,373(86 \cdot 5)$ & $10,452(40 \cdot 4)$ & 12,251 & 340 \\
\hline & Months $37-60$ of IRS & 33,201 & $11,091(33.4)$ & $10,810(97 \cdot 5)$ & $10,399(96 \cdot 2)$ & $1,638(15 \cdot 1)$ & 1,745 & 48 \\
\hline & Months $61-81$ of IRS & 39,711 & $22,318(56 \cdot 2)$ & $22,318(100 \cdot 0)$ & $19,121(85 \cdot 7)$ & $11,155(50 \cdot 0)$ & 11,155 & 531 \\
\hline
\end{tabular}




\begin{tabular}{|c|c|c|c|c|c|c|c|c|}
\hline $\begin{array}{c}\text { MRC } \\
\text { (District) }\end{array}$ & Time period & $\begin{array}{l}\text { Total outpatient } \\
\text { visits, } n\end{array}$ & $\begin{array}{l}\text { Suspected malaria } \\
\text { cases, } n \text { (\% of } \\
\text { outpatient visits) }\end{array}$ & $\begin{array}{l}\text { Tested for malaria, } \\
\mathrm{n} \text { (\% of suspected } \\
\text { malaria cases) }\end{array}$ & $\begin{array}{l}\text { RDT performed } \\
\text { (versus } \\
\text { microscopy), } \mathrm{n} \text { (\% } \\
\text { of tested) }\end{array}$ & $\begin{array}{c}\text { Confirmed malaria } \\
\text { cases, } n(\% \text { of } \\
\text { tested [TPR]) }\end{array}$ & $\begin{array}{c}\text { Confirmed malaria } \\
\text { cases adjusted for } \\
\text { testing }\end{array}$ & $\begin{array}{l}\text { Mean monthly } \\
\text { malaria cases } \\
\text { adjusted for testing }\end{array}$ \\
\hline \multicolumn{9}{|c|}{ IRS SITES } \\
\hline \multirow{2}{*}{$\begin{array}{l}\text { Nagongera HCIV } \\
\text { (Tororo) }\end{array}$} & January-December 2020 & 20,079 & $7,033(35 \cdot 0)$ & $7,031(100 \cdot 0)$ & $5,706(69 \cdot 2)$ & $767(10.9)$ & 767 & 64 \\
\hline & January-October 2021 & 20,405 & $9,827(48 \cdot 2)$ & $9,825(99 \cdot 9)$ & $6,797(83 \cdot 3)$ & $3,706(57 \cdot 7)$ & 3,706 & 371 \\
\hline \multirow{2}{*}{$\begin{array}{c}\text { Amolatar HCIV } \\
\text { (Amolatar) }\end{array}$} & January-December 2020 & 18,995 & $6,360(33.5)$ & $6,349(99 \cdot 8)$ & $5,198(81 \cdot 9)$ & $2,965(46 \cdot 7)$ & 2,969 & 247 \\
\hline & January- October 2021 & 17,230 & $7,311(42 \cdot 2)$ & $7,289(99 \cdot 7)$ & $6,415(88 \cdot 0)$ & $4,198(57 \cdot 6)$ & 4,213 & 421 \\
\hline \multirow{2}{*}{$\begin{array}{l}\text { Dokolo HCIV } \\
\text { (Dokolo) }\end{array}$} & January-December 2020 & 32,042 & $13,984(43 \cdot 6)$ & 13,947 (99.7) & $12,842(92 \cdot 1)$ & $7,110(51 \cdot 0)$ & 7,130 & 594 \\
\hline & January- October 2021 & 25,250 & $10,477(41 \cdot 6)$ & $10,446(99 \cdot 7)$ & $9,117(87 \cdot 2)$ & $4,519(43 \cdot 3)$ & 4,531 & 453 \\
\hline \multirow{2}{*}{$\begin{array}{c}\text { Orum HCIV } \\
\text { (Otuke) }\end{array}$} & January-December 2020 & 11,161 & $6,807(61 \cdot 0)$ & $6,807(100 \cdot 0)$ & $5,672(83 \cdot 3)$ & $2,925(43 \cdot 0)$ & 2,925 & 244 \\
\hline & January- October 2021 & 10,955 & $7,632(70 \cdot 0)$ & $7,632(100 \cdot 0)$ & $3,126(41 \cdot 0)$ & $4,264(55.9)$ & 4,264 & 426 \\
\hline \multirow{2}{*}{$\begin{array}{l}\text { Alebtong HCIV } \\
\text { (Alebtong) }\end{array}$} & January-December 2020 & 19,027 & $8,877(46 \cdot 7)$ & $8,877(100 \cdot 0)$ & $7,763(87 \cdot 5)$ & $2,797(31 \cdot 5)$ & 2,797 & 233 \\
\hline & January- October 2021 & 21,861 & $13,953(63 \cdot 4)$ & $13,953(100 \cdot 0)$ & $11,851(84.9)$ & $8,485(60 \cdot 8)$ & 8,485 & 849 \\
\hline \multicolumn{9}{|c|}{ NON-IRS SITES } \\
\hline \multirow{2}{*}{$\begin{array}{c}\text { Aduku HCIV } \\
\text { (Kwania) }\end{array}$} & January-December 2020 & 26,537 & $19,136(72 \cdot 1)$ & 19,133 (99.9) & $12,770(66 \cdot 7)$ & $12,818(67 \cdot 0)$ & 12,821 & 1,068 \\
\hline & January- October 2021 & 22,196 & $14,220(64 \cdot 1)$ & $14,213(100 \cdot 0$ & $7,308(51 \cdot 4)$ & $7,160(50 \cdot 4)$ & 7,164 & 716 \\
\hline \multirow{2}{*}{$\begin{array}{l}\text { Patongo HCIII } \\
\text { (Agago) }\end{array}$} & January-December 2020 & 21,138 & $18,076(85 \cdot 6)$ & $18,071(99.9)$ & $17,417(96.4)$ & $11,911(65.9)$ & 11,914 & 993 \\
\hline & January- October 2021 & 11,656 & $9,023(77 \cdot 4)$ & $9,023(100 \cdot 0)$ & 7,522 (83.4) & $4,009(44 \cdot 4)$ & 4,009 & 401 \\
\hline \multirow{2}{*}{$\begin{array}{c}\text { Bbaale HCIV } \\
\text { (Kayunga) }\end{array}$} & January-December 2020 & 25,382 & $15,691(61 \cdot 8)$ & 15,591 (99.4) & $11,856(76 \cdot 0)$ & $7,401(47 \cdot 5)$ & 7,450 & 621 \\
\hline & January- October 2021 & 15,266 & $8,185(53 \cdot 6)$ & $8,154(99 \cdot 6)$ & $6,187(75 \cdot 9)$ & $2,502(30 \cdot 7)$ & 2,511 & 251 \\
\hline \multirow{2}{*}{$\begin{array}{l}\text { Lumino HCIII } \\
\text { (Busia) }\end{array}$} & January-December 2020 & 21,885 & $17,783(81 \cdot 2)$ & $17,783(100 \cdot 0)$ & $16,094(90 \cdot 5)$ & $10,409(58.5)$ & 10,409 & 867 \\
\hline & January- October 2021 & 14,354 & $11,018(76 \cdot 8)$ & $11,018(100 \cdot 0)$ & $8,542(77 \cdot 5)$ & $5,435(49 \cdot 3)$ & 5,435 & 544 \\
\hline \multirow{2}{*}{$\begin{array}{l}\text { Apwori HCIII } \\
\text { (Kwania) }\end{array}$} & January-December 2020 & 14,759 & $13,783(93 \cdot 4)$ & $13,781(99 \cdot 9)$ & $12,189(88.5)$ & $9,164(66 \cdot 5)$ & 9,166 & 764 \\
\hline & January- October 2021 & 9,506 & $8,058(84 \cdot 8)$ & $8,058(100 \cdot 0)$ & $8,014(99.5)$ & $2,826(35 \cdot 1)$ & 2,826 & 283 \\
\hline \multirow{2}{*}{$\begin{array}{l}\text { Lira-Kato HCIII } \\
\text { (Agago) }\end{array}$} & January-December 2020 & 20,059 & $17,825(88.9)$ & $17,814(99 \cdot 9)$ & $17,801(99 \cdot 9)$ & $12,670(71 \cdot 1)$ & 12,679 & 1057 \\
\hline & January- October 2021 & 12,064 & $9,715(80 \cdot 5)$ & $9,714(100 \cdot 0)$ & $6,871(70 \cdot 7)$ & $5,313(54 \cdot 7)$ & 5,313 & 531 \\
\hline \multirow{2}{*}{$\begin{array}{l}\text { Morungatuny HCIII } \\
\text { (Amuria) }\end{array}$} & January-December 2020 & 15,053 & 13,964 (92.7) & $13,964(100 \cdot 0)$ & $13,940(99 \cdot 8)$ & $7,698(55 \cdot 1)$ & 7,698 & 642 \\
\hline & January- October 2021 & 10,320 & $9,759(94 \cdot 5)$ & $9,759(100 \cdot 0)$ & $9,758(100 \cdot 0)$ & $3,168(32 \cdot 5)$ & 3,168 & 317 \\
\hline \multirow{2}{*}{$\begin{array}{l}\text { Asamuk HCIII } \\
\text { (Amuria) }\end{array}$} & January-December 2020 & 20,504 & $18,065(88 \cdot 1)$ & $18,045(99.9)$ & $17,633(97 \cdot 7)$ & $10,728(59 \cdot 5)$ & 10,742 & 895 \\
\hline & January- October 2021 & 18,230 & $15,668(85.9)$ & $15,668(100 \cdot 0)$ & 15,387 (98.2) & $6,325(40 \cdot 4)$ & 6,325 & 633 \\
\hline \multirow{2}{*}{$\begin{array}{c}\text { Kapelebyong HCIV } \\
\text { (Kapelebyong) }\end{array}$} & January-December 2020 & 16,975 & $10,720(63 \cdot 2)$ & $10,711(99 \cdot 9)$ & $10,652(99 \cdot 5)$ & $6,215(58 \cdot 0)$ & 6,219 & 518 \\
\hline & January- October 2021 & 15,579 & $7,858(50 \cdot 4)$ & $7,858(100 \cdot 0)$ & 7,849 (99.9) & $2,596(33 \cdot 0)$ & 2,596 & 260 \\
\hline \multirow{2}{*}{$\begin{array}{l}\text { Obalanga HCIII } \\
\text { (Kapelebyong) }\end{array}$} & January-December 2020 & 17,438 & $15,855(90.9)$ & $15,852(99 \cdot 9)$ & $12,833(81 \cdot 0)$ & $9,917(62 \cdot 6)$ & 9,919 & 827 \\
\hline & January- October 2021 & 12,438 & $11,397(91 \cdot 6)$ & $11,397(100 \cdot 0)$ & $9,834(86 \cdot 3)$ & $5,079(41 \cdot 6)$ & 5,079 & 508 \\
\hline
\end{tabular}

